\title{
Pregnancy Outcome among Women with Sickle Cell Disease in a Tertiary Health Institution in Abakaliki: A Retrospective Case-Control Study
}

\author{
Johnbosco Ifunanya Nwafor*, Darlington-Peter Chibuzor Ugoji, Chukwunenye Chukwu Ibo, \\ Blessing Idzuinya Onwe, Victor Jude Uchenna Onuchukwu, Chuka Nobert Obi, Vitus Okwuchukwu Obi
}

Department of Obstetrics and Gynaecology, Alex Ekwueme Federal University Teaching Hospital, Abakaliki, Nigeria

Email: ^nwaforjohnbosco97@gmail.com

How to cite this paper: Nwafor, J.I., Ugoji, D.-P.C., Ibo, C.C., Onwe, B.I., Onuchukwu, V.J.U., Obi, C.N. and Obi, V.O. (2019) Pregnancy Outcome among Women with Sickle Cell Disease in a Tertiary Health Institution in Abakaliki: A Retrospective Case-Control Study. International Journal of Clinical Medicine, 10, 395-403. https://doi.org/10.4236/ijcm.2019.108032

Received: July 6, 2019

Accepted: August 9, 2019

Published: August 12, 2019

Copyright $\odot 2019$ by author(s) and Scientific Research Publishing Inc. This work is licensed under the Creative Commons Attribution International License (CC BY 4.0).

http://creativecommons.org/licenses/by/4.0/

\section{Abstract}

Background: Sickle cell disease (SCD) is associated with an increased risk of medical complications during pregnancy and they constitute a very high-risk group with associated increased maternal and perinatal morbidity and mortality especially in a low resource setting. Objective: To determine the pregnancy outcomes among women with sickle cell disease delivered at Alex Ekwueme Federal University Teaching Hospital, Abakaliki. Materials and methods: This was a 7-year retrospective case-control study undertaken from January 2012 to December 2018 that compared pregnancy outcomes among women with and without haemoglobinopathy in pregnancy managed at Alex Ekwueme Federal University Teaching Hospital, Abakaliki (AEFUTHA). The statistical analysis was done using SPSS version 22. Results: The incidence of SCD in pregnancy was 6.9 per 1000 deliveries. The age distribution of the women ranged from 18 to 45 years of age with a mean of $26.4 \pm 2.4$ years. The incidence of stillbirth was higher in women with HbSS when compared with $\mathrm{HbSC}$ but this difference did not reach statistical significance $(P=0.05)$. Live birth rate was higher in women with HbAA genotype when compared with those with SCD. Caesarean section rate was higher among women with SCD when compared with control (SS versus AA, $\mathrm{P}=0.004$; SC versus AA, $\mathrm{P}<$ 0.0001). Babies of mothers with HbSS and HbSC have significantly lower mean birth weight when compared with those of mothers with HbAA (SS versus AA, $\mathrm{P}=0.0007$; $\mathrm{SC}$ versus $\mathrm{AA}, \mathrm{P}<0.0001)$. Similarly maternal genotype has a significant effect on other adverse fetal outcomes such as Apgar scores $<7$ at 5 minutes and preterm delivery. Women with SCD had higher incidence of pregnancy-induced hypertension and preeclampsia when compared with control. Maternal genotypes have no significant effect on other maternal complications. There was no maternal death in this study. Conclu- 
sion: This study showed that the maternal mortality in SS and SC patients in pregnancy was not different from those of HbAA women in our hospital, although other maternal and fetal outcomes were still poor among women with SCD when compared with women without SCD.

\section{Keywords}

Pregnancy, Outcome, Sickle Cell Disease, Pregnancy, Abakaliki

\section{Introduction}

Sickle cell disease (SCD) is associated with an increased risk of medical complications during pregnancy and they constitute a very high-risk group with associated increased maternal and perinatal morbidity and mortality [1]-[7].

Sickle cell disease is common in the tropics and particularly so among the black race. The incidence in Nigeria is up to $3 \%$ of the population and about $25 \%$ may be carriers of haemoglobinopathies [1]. The haemoglobinopathies commonly encountered in pregnancy in Nigeria are SS or SC [1]. Sickle cell disease is common in present day obstetric practice because the advances in medical care have led to more girls having this condition surviving to childbearing age [2].

Pregnant women with SCD are known to be at high risk of obstetric complications and perinatal mortality as well as sickle cell-related complications [3]. The maternal and fetal complications include antepartum and postpartum painful crises, pulmonary complications, anemia, preeclampsia, eclampsia, premature delivery with associated risks, and intrauterine growth restriction (IUGR). In addition, pregnant women with SCD are at an increased risk of sickle cell crisis, urinary tract infections, gestational diabetes, pneumonia, and anemia [1]-[10].

Studies from developed countries have shown that there is a significant improvement in pregnancy outcome and that these women are able to complete pregnancy successfully if they are given appropriate prenatal care [4]. Unfortunately, no such improvement has yet been observed in sub-Saharan countries, which have the highest prevalence of SCD and reported rates of maternal mortality exceeding 9\% [5] [6] [7] [8] [9]. Lack of adequate management during pregnancy is thought to be the major factor responsible for the poor maternal and fetal outcomes among women with SCD in sub-Saharan Africa compared with developed countries [11].

Currently, there is no Sickle cell clinic dedicated to active management of SCD during pregnancy in Abakaliki. Examining the possible complications in pregnancy associated with SCD may provide insight into the management of SCD pregnancies and it will help to provide for advocacy for establishment of such clinic in the different geopolitical zones of the country. The aim of this study was to identify association between SCD in pregnancy and the occurrence of adverse 
maternal and fetal outcomes at Alex Ekwueme Federal University Teaching Hospital, Abakaliki, Southeast Nigeria. Determining maternal and perinatal outcomes among pregnant women with SCD will highlight the reproductive health burden of SCD on maternal and infant health in Abakaliki, which may contribute to the basis for reducing the maternal and fetal mortality in our locality.

\section{Materials and Method}

This was a 7-year retrospective case-control study undertaken from January 2012 to December 2018 at the Alex Ekwueme Federal University Teaching Hospital, Abakaliki, Ebonyi State, Nigeria. Pregnant women with SCD (HbSS or HbSC genotypes) who received antenatal care and gave birth at the department of obstetrics and gynecology were compared with pregnancies among women without hemoglobinopathies (control). The controls were women without haemoglobinopathies who matched SCD parturients with regards to age, parity and gestational age at delivery. The exclusion criteria were multiple pregnancies, incomplete or unavailable medical records, and presence of co-morbidity such as human immunodeficiency virus (HIV)/acquired immunodeficiency syndrome (AIDS). The hospital numbers of the eligible women during the study period were retrieved from the admission register in the Antenatal ward. Then, the case notes were retrieved from the Medical Records Department of the hospital using the hospital numbers. A proforma containing information on the maternal age, parity, gestational age at delivery and haemoglobin concentration before labour and 48 hour post delivery was used to extract information from the case notes. Data regarding pregnancy outcome (miscarriage, stillbirth, live birth, early neonatal death), mode of delivery (live birth), outcome of live birth (birthweight, Apgar score $<7$ at 5 minutes). In addition, complications such as gestational hypertension (pregnancy-induced hypertension, pre-eclampsia, eclampsia), haemorrhage (antepartum and postpartum), gestational diabetes, retained placenta, uneventful pregnancies, sickle cell-related complications (painful crises, acute chest syndrome, urinary tract infection) and maternal death were also noted. The approval for the study was sought for and obtained from the Research and Ethics Committee of the hospital.

Statistical analysis: The data was entered and analyzed using SPSS Version 22.0 (SPSS Inc., Chicago, IL, USA). Data are presented as mean (standard deviation) or as a percentage with range, as appropriate. The effect of SCD on pregnancy was compared using Pearson Chi-square $\left(\chi^{2}\right)$ or one-way analysis of variance where appropriate to determine statistical differences between the groups of women (SCD and control). Multivariate logistic regression was used to control for confounding factors. A p-value $<0.05$ was considered significant.

\section{Results}

There were 23,450 deliveries during the study period of which 164 (0.69\%) were 
women with SCD. The cases were 98 women with HbSS and 68 with HbSC genotype, whereas the control group was 160 randomly selected women with HbAA with a complete record to match the SCD group in terms of age, gravidity, and parity.

Table 1 showed the socio-demographic and obstetrics characteristics of study participants. The age distribution of the women ranged from 18 to 45 years of age with a mean of $26.4 \pm 2.4$ years. The majority of the women were between 25 and 34 years of age. There were no significant difference in the mean age of women with SCD and the control group $(\mathrm{P}=0.86)$. A majority of the HbSS women (44\%) were nulliparas (58\%) compared with control group $(33.1 \%)$, (P < 0.001). However, there was no significant difference in parity among HbSC and HbAA women. Overall, the mean haemoglobin concentration before labour and 48 hours following delivery were lower among women with SCD when compared with the control group (Mean haemoglobin before labour; $\mathrm{HbAA}=10.6 \pm$ 2.4 versus $\mathrm{HbSS}=8.4 \pm 0.8$ and $\mathrm{HbSC}=9.6 \pm 1.5$; Mean Haemoglobin 48 hours post-delivery; $\mathrm{HbAA}=9.2 \pm 2.8$ versus $\mathrm{HbSS}=6.9 \pm 1.3$ and $\mathrm{HbSC}=8.2 \pm 1.8$ ).

The comparison of pregnancy outcomes between SCD women and HbAA women is shown in Table 2. The incidence of miscarriage was higher among women with HbSS genotype when compared with other genotypes. Four (4.1\%)

Table 1. Characteristics of pregnant women with sickle cell disease (HbSC and HbSS) and control (HbAA).

\begin{tabular}{|c|c|c|c|}
\hline Variable & $\operatorname{HbSS}(\mathrm{N}=98)$ & $\operatorname{HbSC}(\mathrm{N}=66)$ & $\mathrm{HbAA}(\mathrm{N}=160)$ \\
\hline \multicolumn{4}{|l|}{ Age (years) } \\
\hline $18-24$ & $28(28.6)$ & $16(24.2)$ & $47(29.4)$ \\
\hline $25-34$ & $56(57.1)$ & $33(50)$ & $78(48.8)$ \\
\hline$\geq 35$ & $14(14.3)$ & $17(25.8)$ & $35(21.8)$ \\
\hline \multicolumn{4}{|l|}{ Parity } \\
\hline Nulliparous & $44(44.9)$ & $28(42.4)$ & $53(33.1)$ \\
\hline Primiparous & $35(35.7)$ & $23(34.8)$ & $46(28.8)$ \\
\hline Multiparous & $19(19.4)$ & $15(22.8)$ & $61(38.1)$ \\
\hline \multicolumn{4}{|c|}{ Gestational age at delivery } \\
\hline$\leq 27$ & $4(4.1)$ & $0(0)$ & $0(0)$ \\
\hline $28-30$ & $2(2)$ & $1(1.5)$ & $1(0.6)$ \\
\hline $31-32$ & $6(6.1)$ & $4(6.1)$ & $5(3.1)$ \\
\hline $33-34$ & $9(9.2)$ & $6(9.1)$ & $10(6.3)$ \\
\hline $35-37$ & $11(11.2)$ & $14(21.2)$ & $24(15)$ \\
\hline $38-39$ & $56(57.1)$ & $34(51.5)$ & $88(55)$ \\
\hline $40-42$ & $10(10.3)$ & $7(10.6)$ & $32(20)$ \\
\hline \multicolumn{4}{|c|}{$\begin{array}{l}\text { Mean haemoglobin concentration } \\
\text { (g/dl) }\end{array}$} \\
\hline Before labour & $8.4 \pm 0.8$ & $9.6 \pm 1.5$ & $10.6 \pm 2.4$ \\
\hline 48 hour post delivery & $6.9 \pm 1.3$ & $8.2 \pm 1.8$ & $9.2 \pm 2.8$ \\
\hline
\end{tabular}


Table 2. Comparison of pregnancy outcome in sickle cell disease and control (HbAA).

\begin{tabular}{|c|c|c|c|c|c|c|c|c|c|}
\hline \multirow{2}{*}{ Variable } & \multirow{2}{*}{$\mathrm{HbSC}$} & \multirow{2}{*}{ HbSS } & \multirow{2}{*}{ HbAA } & \multicolumn{2}{|c|}{ SS versus SC } & \multicolumn{2}{|c|}{ SS versus AA } & \multicolumn{2}{|c|}{$\mathrm{SC}$ versus $\mathrm{AA}$} \\
\hline & & & & $\chi^{2}$ & P-value & $\chi^{2}$ & P-value & $\chi^{2}$ & P-value \\
\hline Total pregnancies (n) & 66 & 98 & 160 & & & & & & \\
\hline \multicolumn{10}{|l|}{ Pregnancy outcome, $\mathrm{n}(\%)$} \\
\hline Miscarriage & $0(0)$ & $4(4.1)$ & $0(0)$ & - & - & - & - & - & - \\
\hline Stillbirth & $6(9.1)$ & $20(20.4)$ & $2(1.3)$ & 3.79 & 0.05 & 28.6 & 1.43 & 8.41 & 0.004 \\
\hline Live birth & $60(90.9)$ & $74(75.5)$ & $158(98.7)$ & 4.2 & 0.08 & 36.2 & $<0.0001$ & 8.31 & 0.003 \\
\hline Early neonatal death & 2 & 3 & 3 & 0.05 & 0.82 & 0.93 & 0.34 & 0.39 & 0.53 \\
\hline \multicolumn{10}{|l|}{ Mode of delivery (live birth) } \\
\hline Vaginal & $43(71.9)$ & $58(78.4)$ & $145(91.8)$ & 43.81 & 0.54 & 8.26 & 0.004 & 14.81 & $<0.0001$ \\
\hline Caesarean section & $17(28.1)$ & $16(21.6)$ & $13(8.2)$ & & & & & & \\
\hline Outcome of live birth (n) & 60 & 74 & 158 & & & & & & \\
\hline \multicolumn{10}{|l|}{ Birthweight } \\
\hline Mean \pm SD & $2.9 \pm 0.6$ & $2.3 \pm 0.4$ & $3.2 \pm 0.7$ & - & $<0.0001$ & - & 0.0007 & - & $<0.0001$ \\
\hline Weight $<2.5 \mathrm{~kg}, \mathrm{n}(\%)$ & $24(40)$ & $10(13.5)$ & $8(5.1)$ & 12.28 & $<0.0001$ & 5.03 & 0.02 & 42.38 & $<0.0001$ \\
\hline Apgar score $<7$ at 5 minutes & $8(13.3)$ & $6(8.1)$ & $14(8.9)$ & 0.97 & 0.33 & 0.03 & 0.84 & 0.96 & 0.33 \\
\hline \multicolumn{10}{|l|}{ Gestational age at delivery } \\
\hline Preterm (<37 weeks) & $20(33.3)$ & $21(21.4)$ & $19(11.9)$ & 0.38 & 0.54 & 9.45 & 0.002 & 13.44 & $<0.0001$ \\
\hline Mean \pm SD & $37.6 \pm 1.4$ & $37.1 \pm 1.2$ & $38.6 \pm 2.1$ & - & 0.03 & - & $<0.0001$ & - & 0.0008 \\
\hline
\end{tabular}

women had miscarriage among women with HbSS. There were no miscarriages recorded among women with genotypes HbSC and HbAA. More stillbirths occurred in women with haemoglobinopathy when compared with control group. The incidence of stillbirth was higher in women with HbSS when compared with HbSC but this difference did not reach statistical significance $(P=0.05)$. Live birth rate was higher in women with HbAA genotype when compared with those with heamoglobinopathy. There was no significant different in live birth rate among women with HbSS and HbSC $(\mathrm{P}=0.08)$. Mode of delivery differ significantly between women with sickle cell haemoglobinopathy when compared with control (SS versus AA, P = 0.004; SC versus AA, $\mathrm{P}<0.0001$ ). There was no significant difference in the mode of delivery when parturients with HbSS were compare with women with $\operatorname{HbSC}(\mathrm{P}=0.54)$. Maternal genotype has a significant effect on the mean fetal birth weight. Babies of mothers with HbSS and HbSC have significantly lower mean birth weight when compared with those of mothers with $\mathrm{HbAA}$ (SS versus AA, $\mathrm{P}=0.0007$; SC versus AA, $\mathrm{P}<0.0001$ ). Also the birth weight of babies born to women with $\mathrm{HbSS}$ was significantly lower than those of babies born to mothers with HbSC $(P<0.0001)$. Similarly maternal genotype has a significant effect on other adverse fetal outcomes such as Apgar scores $<7$ at 5 minutes and preterm delivery.

Table 3 shows comparison of complications during pregnancy in the three genotypes. There were significant genotype differences in pregnancy-induced 
Table 3. Comparison of pregnancy-associated complications in sickle cell disease and control.

\begin{tabular}{|c|c|c|c|c|c|c|c|c|c|}
\hline \multirow{2}{*}{ Variable } & \multirow{2}{*}{$\mathrm{HbSC}$} & \multirow{2}{*}{ HbSS } & \multirow{2}{*}{ HbAA } & \multicolumn{2}{|c|}{ SS versus SC } & \multicolumn{2}{|c|}{ SS versus AA } & \multicolumn{2}{|c|}{$\mathrm{SC}$ versus $\mathrm{AA}$} \\
\hline & & & & $\chi^{2}$ & P-value & $x^{2}$ & P-value & $\chi^{2}$ & P-value \\
\hline Total pregnancies (n) & 66 & 98 & 160 & & & & & & \\
\hline \multicolumn{10}{|l|}{ Gestational hypertension } \\
\hline Pregnancy-induced hypertension & 9 & 10 & 6 & 0.45 & 0.50 & 4.35 & 0.04 & 7.37 & 0.007 \\
\hline Pre-eclampsia & 14 & 20 & 10 & 0.02 & 0.89 & 11.86 & 0.0005 & 11.02 & 0.001 \\
\hline Eclampsia & 0 & 1 & 0 & - & - & - & - & - & - \\
\hline \multicolumn{10}{|l|}{ Haemorrhage } \\
\hline Antepartum & 3 & 3 & 2 & 0.25 & 0.62 & 1.05 & 0.31 & 2.35 & 0.13 \\
\hline Postpartum & 5 & 7 & 6 & 0.01 & 0.92 & 1.46 & 0.23 & 1.48 & 0.22 \\
\hline Gestational diabetes & 0 & 3 & 2 & 2.06 & 0.15 & 1.05 & 0.31 & 0.83 & 0.36 \\
\hline Retained placenta & 0 & 1 & 0 & - & - & - & - & - & - \\
\hline Uneventful pregnancies & 41 & 34 & 144 & 11.95 & 0.0005 & 86.89 & $<0.0001$ & 22.61 & $<0.0001$ \\
\hline \multicolumn{10}{|l|}{ Sickle cell-related complications } \\
\hline Painful crises & 10 & 29 & 0 & 4.54 & 0.03 & & & & \\
\hline Acute chest syndrome & 6 & 12 & 0 & 0.40 & 0.53 & & & & \\
\hline Urinary tract infection & 12 & 14 & 0 & 0.45 & 0.50 & & & & \\
\hline Maternal death & 0 & 0 & 0 & & & & & & \\
\hline
\end{tabular}

hypertension and pre-eclampsia. Women with haemoglobinopathies have higher incidence of pregnancy-induced hypertension and preeclampsia when compared with control. No SC and AA pregnancies manifested eclampsia compared with one SS. Maternal genotypes have no significant effect on other maternal complications. There was no difference on the incidence of sickle-related clinical events during pregnancy in SC mothers compared SS mothers. This study did not record any maternal death.

\section{Discussion}

The incidence of SCD in pregnancy varies significantly in different parts of the world. The incidence of SCD in pregnancy in this study was 6.9 per 1000 deliveries. This finding was similar to an incidence of 8.7 per 1000 deliveries in Benin, Nigeria [10]. This is higher than reported incidence of 0.95 per 1000 deliveries in Tanzania [11]. Nigeria has a high prevalence of SCD although population data is not available. Data about the incidence of pregnancies in women with SCD are mostly institutional.

It is well established that women with SCD are at increased risk of maternal and fetal complications during pregnancy when compared with healthy women [12] [13] [14] [15] [16]. Studies in low income countries have reported maternal mortality rate of $7 \%-12 \%$ among women with SCD in pregnancy, reflecting limited services and inadequate antenatal care [13]. We report for the first time the outcome of pregnant women with SCD delivering at the Alex Ekwueme 
Federal University Teaching Hospital Abakaliki and compare these outcomes with a comparison group of women with no hemoglobinopathies. Unlike other studies in developing countries, this study indicates that there is significant improvement in the outcome of women with SCD compared with women without hemoglobinopathies. The SCD mortality rate was $0 \%$ of all maternal mortality, which is different from what has been observed in other studies in Africa suggesting that advances in care for patients with SCD have led to improvement in the outcome for women with SCD in pregnancy although other maternal and perinatal outcomes were worse when compared with women with genotype AA.

Pregnancy has been shown to exacerbate sickle cell crises and increase the rate of hospitalization. Recent study indicates that sickle cell crisis occurred in over $50 \%$ of the pregnant women with SCD [14]. However, sickle cell crises were observed in $15 \%$ of women with HbSC and $30 \%$ of those with HbSS in this study, which is consistent with studies conducted in the United States and Ghana [15] [16]. Similarly, the incidence of sickle cell related complication such acute chest syndrome and urinary tract infection were commoner among pregnant women with HbSS when compared with those with HbSC.

The HbSS women were at a greater risk of being anemic when compared with those with HbSC. Anemia is one of the major complications of sickle cell disease and may be caused by hemolysis or trapping of the red blood cells in the spleen [16]. Anemia in pregnancy has been found to be associated with increased risk for preterm premature rupture of membranes, spontaneous preterm labor, preterm delivery, poor intrauterine growth, and low birth weight infants, which in turn results in higher perinatal morbidity and mortality, and a higher infant mortality rate [16].

The caesarean section was more likely to be performed for pregnant women with SCD than for the comparison group. The caesarean is likely to be elective more often in SCD because of fetal compromise and previous history. Closer fetal monitoring and a lower threshold for tolerating abnormal fetal heart rate patterns, may also contribute to this trend. The caesarean section may also be performed as a result of fetal distress, failure of labor to progress, or discretionary repeated need for surgery. However, because of the retrospective nature of this study, differences between elective and emergency caesarean section could not be established.

Overall, pregnancy outcome was worse in women with SCD when compared with those without haemoglobinopathy. However, the findings of this study suggest that pregnancy complications were less in SC disease than in SS disease. This finding was consistent with reports from several studies [1]-[16]. Gestational age at delivery, mode of delivery, live birth rate, and birthweight in SCD pregnancies showed significant difference to AA controls. The outcomes of pregnancy were worse among women with HbSS when compared with HbSC and $\mathrm{HbAA}$. The better pregnancy outcome in women with HbSC is consistent with the behaviour of the SC genotype, which is often mild and may not be diagnosed 
until later in adult life. Although HbSC women had better pregnancy outcome, this study shows that the incidence of sickle cell-related complications did not differ among women with genotype SS and SC. Therefore, it is not yet possible to predict those SC patients who will develop severe complications in pregnancy and it is a good practice to monitor all pregnancies in SCD closely with delivery in hospital.

\section{Conclusion}

In conclusion, this study showed that the maternal mortality in SS and SC patients in pregnancy is not different from those of HbAA women in our hospital, although other maternal and fetal outcomes were still poor when compared with women without SCD. Therefore, preconceptional care and adequate antenatal and postnatal management by a multidisciplinary team and establishment of sickle cell clinic for SCD in pregnancy will help to further improve pregnancy outcome among these women in our facility.

\section{Limitations}

This study has some limitations. Firstly, this is hospital-based study which includes only women that were managed in the hospital but many women deliver in rural areas without reaching health facility so community-based studies are a better tool. Secondly, due to lack of follow up after discharge, the data on neonatal morbidity and mortality as well as maternal outcome for the rest of the puerperium were not available for analysis and finally retrospective nature of study limits its validity.

\section{Conflicts of Interest}

The authors declare no conflicts of interest regarding the publication of this paper.

\section{References}

[1] Olugbenga, A.O. (2018) Managing Sickle Cell Disease in Pregnancy, the Success and the Challenges: Our Experience in a Semi-Urban Tertiary Health-Care Facility, Southwest, Nigeria. Tropical Journal of Obstetrics and Gynaecology, 35, 342-347. https://doi.org/10.4103/TJOG.TJOG_37_18

[2] Wilson, N.O., Ceesay, F.K., Hibbert, J.M., Driss, A., Obed, S.A., Adjei, A.A., et al. (2012) Pregnancy Outcomes among Patients with Sickle Cell Disease at Korle-Bu Teaching Hospital, Accra, Ghana: Retrospective Cohort Study. The American Journal of Tropical Medicine and Hygiene, 86, 936-942. https://doi.org/10.4269/ajtmh.2012.11-0625

[3] Oteng-Ntim, E., Meeks, D., Seed, P.T., Webster, L., Howard, J., Doyle, P. and Chappell, L.C. (2015) Adverse Maternal and Perinatal Outcomes in Pregnant Women with Sickle Cell Disease: Systematic Review and Meta-Analysis. Blood, 125, 3316-3325. https://doi.org/10.1182/blood-2014-11-607317

[4] Rezai, S., Cavallo, G., Gottimuk-Kala, S., Mercado, R. and Henderson, E.C. (2016) Dual Case Reports of Haemoglobin SC Disease in Pregnancy. Obstetrics \& Gyne- 
cology International Journal, 4, Article ID: 00105. https://doi.org/10.15406/ogij.2016.04.00105

[5] Elenga, N., Adeline, A., Balcaen, J., Vaz, T., Calvez, M., Terraz, A., et al. (2016) Pregnancy in Sickle Cell Disease Is a Very High-Risk Situation: An Observational Study. Obstetrics and Gynecology International, 2016, Article ID: 9069054. https://doi.org/10.1155/2016/9069054

[6] Odum, C.U., Anorlu, R.I., Dim, S.I. and Oyekan, T.O. (2002) Pregnancy Outcome in HbSS-Sickle Cell Disease in Lagos, Nigeria. West African Journal of Medicine, 21, 19-23.

[7] Kuo, K., Aviram, A., Merril, E.M. and Caughey, B.A. (2016) Optimal Timing of Delivery for Women with Sickle Cell Disease. American Journal of Obstetrics \& Gynecology, 214, S272-S273. https://doi.org/10.1016/j.ajog.2015.10.542

[8] Omo-Aghoja, I.O. and Okonofua, F.E. (2007) Pregnancy Outcome in Women with Sickle Cell-A Five Year Review. Nigerian Postgraduate Medical Journal, 14, 151-154.

[9] Rahimy, M.C., Gangbo, A., Adjou, R., Deguenon, C., Goussanou, S. and Alihonou, E. (2000) Effect of Active Prenatal Management on Pregnancy Outcome in Sickle Cell Disease in an African Setting. Blood, 96, 1685-1689.

[10] Boafor, T.K., Olayemi, E., Galadanci, N., Hayfron-Benjamin, C., Dei-Adomakoh, Y., Segbefia, C., et al. (2016) Pregnancy Outcome in Women with Sickle-Cell Disease in Low and High Income Countries: A Systematic Review and Meta-Analysis. BJOG: An International Journal of Obstetrics \& Gynaecology, 123, 691-698. https://doi.org/10.1111/1471-0528.13786

[11] Nwabuko, O.C., Okoh, D.A., Iyalla, C. and Omunakwe, H. (2016) Prevalence of Sickle Cell Disease among Pregnant Women in a Tertiary Health Center in South-South Nigeria. Sub-Saharan African Journal of Medicine, 3, 132-136. https://doi.org/10.4103/2384-5147.190843

[12] Ocheni, S., Onah, H.E., Ibegbulam, O.G. and Eze, M.I. (2007) Pregnancy Outcomes in Patients with Sickle Cell Disease in Enugu, Nigeria. Nigerian Journal of Medicine, 16, 227-230.

[13] Ugboma, H.A. and George, I.O. (2015) Sickle Cell Disease in Pregnancy: Maternal and Fetal Outcome in Port Harcourt, Nigeria. British Journal of Medicine and Medical Research, 7, 40-44. https://doi.org/10.9734/BJMMR/2015/11602

[14] Grosse, S.D., Odame, I., Atrash, H.K., Amendah, D.D., Piel, F.B. and Williams, T.N. (2011) Sickle Cell Disease in Africa: A Neglected Cause of Early Childhood Mortality. American Journal of Preventive Medicine, 41, S398-S405. https://doi.org/10.1016/j.amepre.2011.09.013

[15] Graham, R.S., Luana, L.L., Mark, C., Ian, R.B. and Minerva, T. (2004) Outcome of Pregnancy in Homozygous Sickle Cell Disease. Obstetrics \& Gynecology, 103, 1278-1285. https://doi.org/10.1097/01.AOG.0000127433.23611.54

[16] Njamen, T.N., Tolefack, P.N., Ngouadjeu, E., Nguefack, C.T.C. and Nana, C.N. (2018) Pregnancy Outcome among Patients with Homozygous Sickle Cell Disease: Eight Years Retrospective Cohort in a Tertiary Hospital in Sub-Saharan Africa. Archives of Gynecology and Obstetrics, 1, 4-9. 